\title{
SUBCRITICALITY AND GAUGEABILITY OF THE SCHRÖDINGER OPERATOR
}

\author{
Z. ZHAO
}

\begin{abstract}
We investigate a Schrödinger operator $-\Delta / 2+V$ in $R^{d} \quad(d \geq 3)$ with a potential $V$ in the class $K_{d}$ satisfying a similar Kato condition at infinity, and prove an equivalence theorem connecting various conditions on subcriticality, strong positivity and gaugeability of the operator.
\end{abstract}

\section{INTRODUCTION}

We consider the Schrödinger operator $H=-\Delta / 2+V$ in $R^{d} \quad(d \geq 3)$ from three different aspects: criticality or subcriticality, strong positivity, and gaugeability. In each aspect, we present several conditions on $V$ (or $H$ ). The purpose of this paper is to prove that all these conditions in the three aspects are equivalent for a large class of potentials $V$.

We first assume that the potential $V$ is in Kato class $K_{d}$, i.e. $V$ is a Borel measurable function in $R^{d}$ satisfying

$$
\lim _{r \downarrow 0}\left[\sup _{x \in R^{d}} \int_{|y-x| \leq r} \frac{|V(y)|}{|y-x|^{d-2}} d y\right]=0,
$$

or more generally, $V$ is in $K_{d}^{\text {loc }}$ i.e., for any ball $B, 1_{B} V \in K_{d}$, where $1_{B}$ denotes the indicator function of $B$.

In $\S 2$, we define a new subclass $K_{d}^{\infty}$ of $K_{d}$ and show that $K_{d}^{\infty}$ contains $K_{d} \cap L^{1}\left(R^{d}\right)$ and any potentials behaving like $c|x|^{-\rho} \quad(\rho>2)$ near infinity. In $\S 4$, we shall prove the main equivalence theorem for any potential $V$ in $K_{d}^{\infty}$ after presenting a sequence of preliminary lemmas and propositions in $\S 3$.

In the rest of this section we describe these conditions in the three aspects respectively and discuss the key ideas which connect them.

(i) Criticality or subcriticality: There have been various notions for criticality. One of them, perhaps the most intuitive one, is that $-\Delta / 2+V \geq 0$ but for any essentially nonzero function $q \leq 0,-\Delta / 2+V+q \nsucceq 0$. Under the assumption $H \geq 0$, if any notion for criticality is proposed then the opposite condition will be for subcriticality. Therefore in the following we only consider the subcritical condition. For instance, subcriticality corresponds to the idea described above

Received by the editors January 30, 1991 and, in revised form, August 13, 1990.

1980 Mathematics Subject Classification (1985 Revision). Primary 81C20; Secondary 60J65, $81 \mathrm{C} 12,81 \mathrm{C} 35$.

Key words and phrases. Schrödinger operators, Brownian motion, subcriticality, Feynman-Kac integral. 
is that the operator $H \geq 0$ allows a "small" perturbation and still keeps its nonnegativity. The precise notions for this are conditions (a) and (a1)-(a3) in MT, here and below, MT stands for the main theorem in $\S 4$.

There have been two other notions of subcriticality. (Simon [S1]) there exists a $\beta>0$ such that $-\Delta / 2+(1+\beta) V \geq 0$ ((b) in MT); and (Murata [M2]) there exists a positive Green function $G^{V}(\cdot, \cdot)$ for $H((\mathrm{c})$ in MT).

Some implications and equivalences among these subcritical conditions have been proved under different assumptions on $V$. See [A1, M1, Pi]. A Green function approach for the Schrödinger operators has been given in [H-Z].

(ii) Strong positivity: By the Allegretto-Piepenbrink theory (see [S2]) for $V \in$ $K_{d}^{\text {loc }}, H=-\Delta / 2+V \geq 0$ if and only if there exists a positive solution $u>0$ of $H u=0$ in $R^{d}$. In view of this, one may expect to use the existence of some strongly positive solution $u$ to characterize the subcriticality, for instance, the existence of a solution $u \geq c>0$ of $H u=0$ (see (d), (d1), and (d2) in MT).

(iii) Gaugeability: It is known that the probabilistic counterpart of the Schrödinger operator $-\Delta / 2+V$ is the Feynman-Kac path integral. In this paper we consider the stopped Feynman-Kac integral in the following form:

$$
\mathbb{E}^{x}\left[\exp \left(-\int_{0}^{\xi} V(X s) d s\right)\right],
$$

where $\left\{X_{s}\right\}$ denotes a Markov process in $R^{d}$ with its lifetime $\xi$ and $\mathbb{E}^{x}$ denotes the expectation over the paths starting with $x \in R^{d}$. We take the Markov process $\left\{X_{s}\right\}$ as the standard Brownian motion in $R^{d}$ and the $y$-conditioned Brownian motion for a point $y$ in $R^{d}$ (i.e., $G(y, \cdot)$-process of Doob type see [D]), respectively and use the expectation $E^{x}$ and $E_{y}^{x}$ to distinguish them. We define the gauge and the conditional gauge respectively by

$$
u_{0}(x)=E^{x}\left[\exp \left(-\int_{0}^{\infty} V(X s) d s\right)\right]
$$

and

$$
u_{0}(x, y)=E_{y}^{x}\left[\exp \left(-\int_{0}^{\xi} V(X s) d s\right)\right] .
$$

The gaugeability means the finiteness or boundedness of these quantities (see (e), (e1), (f), and (fl) in MT). In recent years there has been an intensive study on the equivalence of finiteness and boundedness of the gauge and the conditional gauge mainly for a bounded domain in $R^{d}$ (see [Ch-R, C-F-Z, F, $\mathrm{Z}-\mathrm{Z} 3]$ ). In this paper we prove these equivalence for the whole space $R^{d}$ and a potential $V \in K_{d}^{\infty}$ in a similar manner. This part is a continuation of the previous study.

The essential part of the main result lies in the connection between the three aspects. An equality given in [Z3]

$$
\frac{G^{V}(x, y)}{G(x, y)}=E_{y}^{x}\left[\exp \left(-\int_{0}^{\xi} V(X s) d s\right)\right],
$$

reveals a connection between the existence of a positive $G^{V}(\cdot, \cdot)$ and the finiteness of the conditional gauge. But we need a key assertion as a critical linkage among these conditions. 
For $V \in K_{d}^{\infty}$ and a large number $A>0$, we define a shuttle time $\sigma$ for any Brownian path starting at a point on $\partial B(0, A)$ as the first time returning to $\partial B(0, A)$ after hitting $\partial B(0,2 A)$, and a shuttle operator $S_{V}$ in the Banach space $C(\partial B(0, A))$ :

$$
S_{V} f(x)=E^{x}\left[\sigma<\infty ; \exp \left(-\int_{0}^{\sigma} V(X s) d s\right) f(X(\sigma))\right] .
$$

Put

$$
\lambda(V)=\lim _{n \rightarrow \infty} \sqrt[n]{\left\|\left(S_{V}\right)^{n}\right\|}
$$

which is a higher dimensional analogy of the quantity $u(a, b) u(b, a)$ given in [Ch] and [Ch-V].

Introducing the shuttle operator and its spectral radius $\lambda(V)$ is the key idea in connecting the seemingly different conditions. In fact we add a new equivalent condition on $V$ into the list as a central linkage: $\lambda(V)<1$, (see $(\mathrm{g})$ in MT).

For one dimensional case $(d=1)$, in a joint paper with F. Gesztesy [G-Z], we proved the equivalence theorem of subcriticality for the general Sturm-Liouville operators, which becomes an impoitant motivation of this paper. For $d=2$, if we consider a transient domain like $B_{r}^{*}=\left\{x \in R^{2}:|x|>r\right\} \quad(r>0)$, then we can extend the main result to this transient domain, but if we also consider the whole space $R^{2}$, then there is an essential difference with the case $d \geq 3$ due to the recurrence of $R^{2}$. A conjecture may be proposed: $-\Delta / 2+t V$ in $R^{2}$ is critical for each $t \in[0,1]$ (or only for $t=t_{1}$ and $t_{2}, 0<t_{1}<t_{2}$ ) if and only if $V \equiv 0$ a.e. in $R^{2}$. The same statement in the one dimensional case has been proved in $[\mathrm{G}-\mathrm{Z}]$.

\section{Class $K_{d}^{\infty}$ AND BASIC NOtIONS}

We assume that the dimension $d \geq 3$. For any domain $D \subseteq R^{d}, L^{p}(D)$ $(1 \leq p \leq \infty)$ are relative to the Lebesgue measure and put

$$
\mathscr{B}(D)=\{\text { all Borel measurable functions in } D\} \text {. }
$$

For any compact set $\Lambda$ in $R^{d}, C(\Lambda)$ denotes the Banach space of all continuous functions on $\Lambda$ with the maximum norm.

The basic classes of potentials are given by

$$
K_{d}=\left\{V \in \mathscr{B}\left(R^{d}\right): \lim _{a \downarrow 0}\left[\sup _{x \in R^{d}} \int_{|y-x| \leq a} \frac{|V(y)|}{|y-x|^{d-2}} d y\right]=0\right\},
$$

and

$$
K_{d}^{\text {loc }}=\left\{V \in \mathscr{B}\left(R^{d}\right): \forall \rho>0,1_{B(0, \rho)} V \in K_{d}\right\},
$$

(see $[\mathrm{A}-\mathrm{S}]$ or $[\mathrm{S} 2])$.

We now introduce a new function class

\section{Definition 1.}

$$
K_{d}^{\infty}=\left\{V \in K_{d}^{\text {loc }}: \lim _{A \uparrow \infty}\left[\sup _{x \in R^{d}} \int_{|y| \geq A} \frac{|V(y)|}{|y-x|^{d-2}} d y\right]=0\right\} .
$$


Proposition 1. $K_{d} \cap L^{1}\left(R^{d}\right) \subseteq K_{d}^{\infty} \subseteq K_{d}$.

Proof. Suppose $V \in K_{d} \cap L^{1}\left(R^{d}\right)$. For any $\varepsilon>0$, there is an $a>0$ such that

$$
\sup _{x \in R^{d}} \int_{|y-x| \leq a} \frac{|V(y)|}{|y-x|^{d-2}} d y<\frac{\varepsilon}{2} .
$$

Since $V \in L^{1}\left(R^{d}\right)$, there is an $A>0$ such that

Then we have for each $x \in R^{d}$,

$$
\int_{|y| \geq A}|V(y)| d y<a^{d-2} \frac{\varepsilon}{2}
$$

$$
\begin{aligned}
\int_{|y| \geq A} \frac{|V(y)|}{|y-x|^{d-2}} d y & \leq \int_{|y-x| \leq a} \frac{V(y)}{|y-x|^{d-2}} d y+\frac{1}{a^{d-2}} \int_{|y| \geq A}|V(y)| d y \\
& \leq \frac{\varepsilon}{2}+\frac{1}{a^{d-2}}\left(a^{d-2} \frac{\varepsilon}{2}\right)=\varepsilon .
\end{aligned}
$$

So $V \in K_{d}^{\infty}$.

The inclusion $K_{d}^{\infty} \subseteq K_{d}$ follows from

$$
\begin{aligned}
\{(x, y):|y-x| \leq a\} \subseteq & \{(x, y):|y-x| \leq a \text { and }|x| \leq A+1\} \\
& \cup\{(x, y):|y| \geq A\}, \quad \text { for } 0<a \leq 1 .
\end{aligned}
$$

Proposition 2. For $\rho>2,\left\{V \in K_{d}: V(x)=O\left(|x|^{-\rho}\right)\right.$ as $\left.|x| \rightarrow \infty\right\} \subseteq K_{d}^{\infty}$.

Proof. Since $\rho>2$, we can choose a number $p>1$ such that $d / 2>p>d / \rho$. This implies that $\rho p>d$ and $(d-2) p /(p-1)>d$.

For any $\varepsilon>0$, since $V \in K_{d}, \exists a>0$ such that

$$
\sup _{x} \int_{|y-x| \leq a} \frac{|V(y)|}{|y-x|^{d-2}} d y \leq \varepsilon .
$$

We suppose that $\exists c>0$ such that for sufficiently large $A$, when $|y| \geq A$, $|V(y)| \leq c /|y|^{\rho}$. Then by Hölder's inequality, for each $x \in R^{d}$,

$$
\begin{gathered}
\int_{|y| \geq A} \frac{|V(y)|}{|y-x|^{d-2}} d y \leq \varepsilon+c \int_{\substack{|y| \geq A \text { and } \\
|y-x| \geq a}} \frac{d y}{|y-x|^{d-2}|y|^{\rho}} \\
\leq \varepsilon+c\left(\int_{|y| \geq A} \frac{d y}{|y|^{\rho p}}\right)^{1 / p}\left(\int_{|y-x| \geq a} \frac{d y}{|y-x|^{(d-2) p /(p-1)}}\right)^{(p-1) / p} \\
\quad=\varepsilon+c\left(\int_{|y| \geq A} \frac{d y}{|y|^{\rho p}}\right)^{1 / p}\left(\int_{|y| \geq a} \frac{d y}{|y|^{(d-2) p /(p-1)}}\right)^{(p-1) / p} .
\end{gathered}
$$

Therefore we obtain

So we conclude $V \in K_{d}^{\infty}$.

$$
\varlimsup_{A \uparrow \infty}\left[\sup _{x \in R^{d}} \int_{|y| \geq A} \frac{|V(y)|}{|y-x|^{d-2}} d y\right] \leq \varepsilon .
$$

For $V \in K_{d}^{\infty}$, put

$$
\|V\| \mid \|=\sup _{x \in R^{d}} \int_{R^{d}} \frac{|V(y)|}{|y-x|^{d-2}} d y .
$$

Obviously, we have $\|V\| \mid<\infty$. In fact, $\left(K_{d}^{\infty},\|\cdot\| \|\right)$ forms a Banach space, but we do not need this point later.

It is easy to verify the following proposition by definition: 
Proposition 3. Let $V \in \mathscr{B}\left(R^{d}\right)$. Then we have

(i) $V \in K_{d}^{\text {loc }}$ if and only if for any bounded domain $D$ in $R^{d}$, the family $\left\{|V(y)| /|x-y|^{d-2}, x \in D\right\}$ is uniformly integrable in $D$.

(ii) $V \in K_{d}^{\infty}$ if and only if the family $\left\{|V(y)| /|x-y|^{d-2}, x \in R^{d}\right\}$ is uniformly integrable in $R^{d}$.

Let $\left\{X_{t}: t \geq 0\right\}$ be the Brownian motion in $R^{d}$ on the probability space $(\Omega, \mathscr{F}, P)$ and let $P^{x}$ and $E^{x}$ denote the probability and the expectation over the Brownian paths starting with $x \in R^{d}$, respectively. For any $A \in \mathscr{F}$ and a positive measurable function $f$ on $\Omega$, we put

$$
E^{x}[A ; f]=\int_{A} f(\omega) P^{x}(d \omega)
$$

For $V \in K_{d}$, put

$$
e_{V}(t)=\exp \left[-\int_{0}^{t} V(X s) d s\right], \quad 0 \leq t \leq \infty .
$$

For any domain $D$ in $R^{d}(d \geq 3)$, let $G_{D}(x, y)$ be the Green function in $D$ corresponding to $-\Delta / 2$. Then for each $y \in D$, we can define the conditional $y$-process in $D \backslash\{y\}$ by the transition density: (see [D, Z3])

$$
\begin{aligned}
& p_{y, D}(t, x, w)=G_{D}(x, y)^{-1} p_{D}(t, x, w) G_{D}(w, y), \\
& \text { for } t>0, x, w \in D \backslash\{y\},
\end{aligned}
$$

where $p_{D}$ is the transition density for the killed Brownian motion outside $D$ (see [P-S]). We shall use $P_{y, D}^{x}$ and $E_{y, D}^{x}$ to denote the probability and the expectation over the $y$-conditioned paths in $D$ starting with $x$, respectively. If $D=R^{d}$, then we shall omit $D$, so they become $P_{y}^{x}$ and $E_{y}^{x}$.

We mainly consider the following subdomains of $R^{d}$ : for $r>0$,

$$
B_{r}=B(0, r)=\left\{x \in R^{d}:|x|<r\right\},
$$

and

$$
B_{r}^{*}=\left\{x \in R^{d}:|x|>r\right\} .
$$

For each $r>0$, the Green function and the Poisson kernel of $B_{r}$ and $B_{r}^{*}$ corresponding to $-\Delta / 2$ have the same form. Let $D_{r}$ be either $B_{r}$ or $B_{r}^{*}$. Then

$$
G_{D_{r}}(x, y)=\frac{c_{d}}{|x-y|^{d-2}}-\frac{c_{d} r^{d-2}}{|y|^{d-2}\left|y^{*}-x\right|^{d-2}}, \quad x, y \in D_{r},
$$

where $y \neq 0, y^{*}=r^{2} y /|y|^{2}$ and $c_{d}=\Gamma\left(\frac{d}{2}-1\right) / 2 \pi^{d / 2}$;

$$
K_{D_{r}}(x, z)=c_{d}(d-2) \frac{\left.\left|r^{2}-\right| x\right|^{2} \mid}{r|x-z|^{d}}, \quad x \in D_{r}, \quad z \in \partial D_{r} .
$$

Remark. When $D_{r}=B_{r}^{*}$, the Poisson kernel $K_{D_{r}}(x, z)$ only represents the harmonic function $h$ with $\lim _{|x| \rightarrow \infty} h(x)=0$. When $D=R^{d}$, we also omit $R^{d}$ i.e., $G(x, y)=c_{d} /|x-y|^{d-2}$.

The following crucial inequality called the $3 G$ estimate (see [Z1] when $D_{r}=$ $B_{r}$ ) can be proved by the same elementary argument as that in [Z1] when $D_{r}=B_{r}^{*}$. 
Proposition 4. For any $r>0$ and any $x, y, z$ in $D_{r}$, we have

$$
\frac{G_{D_{r}}(x, y) G_{D_{r}}(y, z)}{G_{D_{r}}(x, z)} \leq C(d)\left(|x-y|^{2-d}+|y-z|^{2-d}\right),
$$

where

$$
C(d)=2^{d+3}(d-2)^{2} c_{d} .
$$

We end this section with a definition of nonnegativity of the operator $H$.

Definition 2. For $V \in K_{d}^{\text {loc }}, H \equiv-\Delta / 2+V \geq 0$ iff for any $\varphi \in C_{c}^{\infty}\left(R^{d}\right)$,

$$
\frac{1}{2} \int_{R^{d}} \nabla \varphi \cdot \nabla \varphi d x+\int_{R^{d}} V \varphi^{2} d x \geq 0,
$$

where $C_{c}^{\infty}\left(R^{d}\right)=\left\{\right.$ all infinitely differentiable functions in $R^{d}$ with a compact support\}.

Remark. This is equivalent to the condition: Spectrum of $H \subseteq[0, \infty)$.

\section{Preliminary lemmas and propositions}

We start with a probabilistic characterization of nonnegativity of $H$. Recall the notation $e_{V}(\cdot)$ given in (10) and define the exit time of a Markov process $X$ from a domain $D$ by

$$
\begin{aligned}
\tau_{D} & =\inf \left[t<\xi: X_{t} \notin D\right], & & \text { if the set in the bracket is nonempty, } \\
& =\xi, & & \text { if the set in the bracket is empty, }
\end{aligned}
$$

where $\xi$ is the lifetime of $X$.

Theorem 1. Let $V \in K_{d}^{\text {loc }}$. Then $H=-\Delta / 2+V \geq 0$ if and only if for any $r>0$,

$$
E^{x}\left[e_{V}\left(\tau_{B_{r}}\right)\right] \not \equiv \infty \text { in } B_{r} .
$$

Proof. If $-\Delta / 2+V \geq 0$ then by the Allegretto-Piepenbrink theory (see Theorem C.8.1 in [S2] or Theorem 5 in [Z4]), there exists a positive and continuous solution $u>0$ of $H u=0$ in $R^{d}$. For any $r>0$, we have $\inf _{x \in B_{r}} u(x) \geq$ $\inf _{x \in \bar{B}_{r}} u(x)>0$. Thus by Theorem 4 in [Z4], (17) holds.

Conversely, if (17) holds for each $r>0$, then by Theorem 4 in [Z4], all eigenvalues of $-\Delta / 2+V$ in $B_{r}$ are larger than zero, i.e.,

$$
\operatorname{Spec}\left[\left.(-\Delta / 2+V)\right|_{B_{r}}\right] \subseteq(0, \infty) .
$$

For any $\varphi$ in $C_{c}^{\infty}\left(R^{d}\right)$, we can find $r>0$ such that $\operatorname{supp}(\varphi) \subseteq B_{r}$. Then by (18) we have

$$
\frac{1}{2} \int_{R^{3}} \nabla \varphi \cdot \nabla \varphi d x+\int_{R^{d}} V \varphi^{2} d x=\frac{1}{2} \int_{B_{r}} \nabla \varphi \cdot \nabla \varphi d x+\int_{R^{d}} V \varphi^{2} d x \geq 0 .
$$

This shows $H=-\Delta / 2+V \geq 0$ by the Definition 2 .

Proposition 5. Suppose $V \in K_{d}^{\text {loc }}$ and $H=-\Delta / 2+V \geq 0$. Then for any bounded $C^{2}$ domain $D$ in $R^{d}$, there exists a jointly continuous Poisson kernel 
$K_{D}^{V}(x, z)>0,(x, z) \in D \times \partial D$ for the operator $H$, i.e., for any $f \in C(\partial D)$ and $x \in D$, we have

$$
\int_{\partial D} K_{D}^{V}(x, z) f(z) \sigma(d z)=E^{x}\left[e_{V}\left(\tau_{D}\right) f\left(X\left(\tau_{D}\right)\right)\right] .
$$

Proof. The proposition follows from Theorem 1 and Corollary 2 in [Z3].

We now state a crucial lemma in a general situation, which can be proved by the same argument as that in [AS, Theorem 1.2].

Lemma 1 (Khasminskii's lemma). Suppose $D$ is a domain in $R^{d}, V \in \mathscr{B}(D)$, $\left\{X_{t}\right\}$ is a Markov process in $D$ and $\tau$ is either a constant time or an exit time. If

$$
\sup _{x \in D} \mathbb{E}^{x}\left[\int_{0}^{\tau}\left|V\left(X_{s}\right)\right| d s\right] \leq \alpha<1
$$

then

$$
\sup _{x \in D} \mathbb{E}^{x}\left[\exp \left(\int_{0}^{\tau}\left|V\left(X_{s}\right)\right| d s\right)\right] \leq \frac{1}{1-\alpha} .
$$

Note that we use $\mathbb{E}^{x}$ for the general process.

Lemma 2. Under the same assumptions as in Lemma 1, let $x \in D, A \in \mathscr{F}$ with $\mathbb{P}^{x}(A) \geq c_{1}>0$, and $\mathbb{E}^{x}\left[\int_{0}^{\tau}\left|V\left(X_{s}\right)\right| d s\right] \leq c_{2}$.

Then we have

$$
\mathbb{E}^{x}\left[A ; e_{V}(\tau)\right] \geq c_{1} \exp \left(-c_{2} / c_{1}\right) .
$$

Proof. It is easily done by using the Jensen's inequality for $\mathbb{E}^{x}(\cdot \mid A)$.

Lemma 3. Let $D$ be a ball $B=B(w, l)$ in $R^{d}$ or $B_{r}^{*}(r>0)$ or $R^{d}$ itself, and let $U$ be a subdomain of $D$ satisfying

$$
\left\|1_{U} V\right\|<\frac{1}{2 C(d)},
$$

where $C(d)$ is given in (15). Then for all $x$ and $y$ in $D$ with $x \neq y$ we have

$$
E_{y, D}^{x}\left[\exp \left(\int_{0}^{\tau_{U}}\left|V\left(X_{s}\right)\right| d s\right)\right] \leq \frac{1}{1-2 C(d)\left|\| 1_{U} V\right| \mid} .
$$

Proof. For each $x, y \in D$ with $x \neq y$, by Proposition 4 and Fubini's theorem we have

$$
\begin{aligned}
E_{y, D}^{x} & {\left[\int_{0}^{\tau_{U}}\left|V\left(X_{s}\right)\right| d s\right]=\int_{0}^{\infty} E_{y, D}^{x}\left[s<\tau_{U} ;\left|V\left(X_{s}\right)\right|\right] d s } \\
& =G_{D}(x, y)^{-1} \int_{0}^{\infty} E^{x}\left[s<\tau_{U} ; G_{D}\left(X_{s}, y\right)\left|V\left(X_{s}\right)\right|\right] d s \\
& \leq G_{D}(x, y)^{-1} \int_{U} G_{D}(x, w) G_{D}(w, y)|V(w)| d w \\
& \leq C(d)\left[\int_{U} \frac{|V(w)|}{|x-w|^{d-2}} d w+\int_{U} \frac{|V(w)|}{|y-w|^{d-2}} d s\right] \\
& \leq 2 C(d)|| 1_{U} V \mid \| .
\end{aligned}
$$

Thus (24) follows from Lemma 1 and (25). 
Now we assume $V \in K_{d}^{\infty}(d \geq 3)$. By Definition 1, there exists a number $A>0$ such that

$$
\| 1_{B_{A}^{*}} V|| \mid=\sup _{x \in R^{d}} \int_{|y|>A} \frac{|V(y)|}{|y-x|^{d-2}} d y<\frac{1}{4 C(d)} .
$$

Since $K_{d}^{\infty} \subseteq K_{d}$ (Proposition 1), by the definition of $K_{d}$ given in (6), there exists a number $a$ with $0<a \leq A$ such that for any ball $B(w, r)$ with $0<r \leq a$,

$$
\left\|1_{B(w, r)} V\right\|=\sup _{x \in R^{d}} \int_{B(w, r)} \frac{|V(y)|}{|y-x|^{d-2}} d y<\frac{1}{4 C(d)} .
$$

Fix the two numbers $A$ and $a$ which depend on $d$ and $V$ only.

In the following, we assume

$$
\sup _{x \in B_{2 A}} E^{x}\left[e_{V}\left(\tau_{B_{2 A}}\right)\right]<\infty
$$

The next lemma follows from Lemma 1 and (26).

\section{Lemma 4.}

Put

$$
\sup _{x \in R^{d}} E^{x}\left[\exp \left(\int_{0}^{\tau_{B_{A}^{*}}}\left|V\left(X_{s}\right)\right| d s\right)\right] \leq 2 .
$$

$$
u_{0}(x)=E^{x}\left[e_{V}(\infty)\right]=E^{x}\left[\exp \left(-\int_{0}^{\infty} V\left(X_{s}\right) d s\right)\right], \quad x \in R^{d} .
$$

Theorem 2. $u_{0}(x) \not \equiv \infty$ in $R^{d}$ if and only if $u_{0}(x)$ is bounded in $R^{d}$. If so, then $u_{0}$ is a continuous solution of $H u=0$ in $R^{d}$.

Proof. Since for any ball $B$ and $x \in B$, we have

$$
\begin{aligned}
u_{0}(x) & =E^{x}\left[e_{V}\left(\tau_{B}\right) E^{X\left(\tau_{B}\right)}\left[e_{V}(\infty)\right]\right] \\
& =E^{x}\left[e_{V}\left(\tau_{B}\right) u_{0}\left(X\left(\tau_{B}\right)\right)\right] .
\end{aligned}
$$

Then by an estimate given by Theorem 1 in [Z1] and a chain argument, it shows that if there is one point $x_{0} \in R^{d}$ with $u_{0}\left(x_{0}\right)<\infty$ then $u_{0}$ is bounded on any compact set, in particular we have $M=\sup _{|x| \leq A} u_{0}(x)<\infty$. Now for each $x \in B_{A}^{*}$,

$$
\begin{aligned}
u_{0}(x)= & E^{x}\left[\tau_{B_{A}^{*}}=\infty, e_{V}\left(\tau_{B_{A}^{*}}\right)\right] \\
& +E^{x}\left[\tau_{B_{A}^{*}}<\infty ; e_{V}\left(\tau_{B_{A}^{*}}\right) u_{0}\left(X\left(\tau_{B_{A}^{*}}\right)\right)\right] \\
\leq & (M+1) E^{x}\left[e_{V}\left(\tau_{B_{A}^{*}}\right)\right] \leq 2(M+1) .
\end{aligned}
$$

The last inequality is due to Lemma 4 . This shows that $u_{0}$ is bounded in $R^{d}$.

Now suppose that $C \equiv \sup _{x \in R^{d}} u_{0}(x)<\infty$. Then we have

$$
\int_{R^{d}} G(x, y)|V(y)| u_{0}(y) d y \leq C c_{d}|\|V \mid\|<\infty .
$$

By the Fubini's theorem together with (31) and the piecewise integration, we get

$$
\begin{aligned}
\int_{R^{d}} G(x, y) V(y) u_{0}(y) d y & =E^{x}\left[\int_{0}^{\infty} V\left(X_{t}\right) E^{X(t)}\left[e_{V}(\infty)\right] d t\right] \\
& =E^{x}\left\{\int_{0}^{\infty} V\left(X_{t}\right) \exp \left[-\int_{t}^{\infty} V\left(X_{s}\right) d s\right]\right\} \\
& =E^{x}\left[1-e_{V}(\infty)\right]=1-u_{0}(x) .
\end{aligned}
$$


Applying $\Delta / 2$ on both sides of (32) we obtain $-\Delta / 2 u_{0}(x)+V(x) u_{0}(x)=$ 0 , i.e., $u_{0}$ is a distributional solution of $H u=0$. By Proposition 3(ii), $\left\{G(x, y)|V(y)| u_{0}(y): x \in R^{d}\right\}$ is uniformly integrable. So the continuity of $u_{0}(x)$ follows from (32).

Lemma 5. There exists a joint continuous Poisson kernel $K_{B_{A}^{*}}^{V}(x, z)>0$ for $H$ on $B_{A}^{*} \times \partial B_{A}^{*}$ with the estimate:

$$
K_{B_{A}^{*}}^{V}(x, z) \leq 2(d-2) c_{d} \frac{|x|^{2}-A^{2}}{A|x-z|^{d}} .
$$

Remark. Poisson kernel here is for representing the solution $u$ of $H u=0$ with $\lim _{|x| \rightarrow \infty} u(x)=0$.

Proof. By (24) in Lemma 3 with $D=U=B_{A}^{*}$ and condition (26) we have

$$
\sup _{(x, y) \in B_{A}^{*} \times \bar{B}_{A}^{*}} E_{y, B_{A}^{*}}^{x}\left[e_{V}\left(\tau_{B_{A}^{*}}\right)\right] \leq 2 .
$$

By Proposition 3(ii), (34) and the method similar to that in [Z3], we have that $(x, z) \rightarrow E_{z, B_{A}^{*}}^{x}\left[e_{V}\left(\tau_{B_{A}^{*}}\right)\right]$ is joint continuous on $B_{A}^{*} \times \partial B_{A}^{*}$ and

$$
K_{B_{A}^{*}}^{V}(x, z) / K_{B_{A}^{*}}(x, z)=E_{z, B_{A}^{*}}^{x}\left[e_{V}\left(\tau_{B_{A}^{*}}\right)\right] \text {. }
$$

Thus (33) follows from (13), (34) and (35).

For each $r$ in $(2 A, \infty]$, put $J_{A, r}=\left\{y \in R^{d}: A<|y|<r\right\}$ (note: $J_{A, \infty}=$ $\left.B_{A}^{*}\right)$. We define a stopping time:

$$
\sigma_{r}=\tau_{B_{2 A}}+\tau_{J_{A, r}} \circ \theta_{\tau_{B_{2 A}}}
$$

and an operator in the Banach space $C\left(\partial B_{A}\right)$ :

$$
\begin{gathered}
S_{V, r} f(z)=E^{z}\left[\sigma_{r}<\infty \text { and } X\left(\sigma_{r}\right) \in \partial B_{A} ; e_{V}\left(\sigma_{r}\right) f\left(X\left(\sigma_{r}\right)\right)\right], \\
z \in \partial B_{A}, f \in C\left(\partial B_{A}\right) .
\end{gathered}
$$

Lemma 6. For each $r \in(2 A, \infty], S_{V, r}$ is an integral operator in $C\left(\partial B_{A}\right)$ with a continuous kernel $\Phi_{r}(\cdot, \cdot)$ :

$$
S_{V, r} f(z)=\int_{\partial B_{A}} \Phi_{r}(z, w) \sigma(d w),
$$

where

$$
\Phi_{r}(z, w)=\int_{\partial B_{2 A}} K_{B_{2 A}}^{V}(z, u) K_{J_{A, r}}^{V}(u, w) \sigma(d u),
$$

$$
(z, w) \in \partial B_{A} \times \partial B_{A},
$$

and $\sigma(\cdot)$ is the area measure.

Proof. In the following, for any closed set $\Lambda$ in $R^{d}, T_{\Lambda}$ denotes the first hitting time on $\Lambda$. By the strong Markov property, we have for any $f \in C\left(\partial B_{A}\right)$,

$$
\begin{aligned}
S_{V, r} f(z)= & E^{z}\left\{e _ { V } ( \tau _ { B _ { 2 A } } ) E ^ { X ( \tau _ { B _ { 2 A } } ) } \left[T\left(\partial B_{A}\right)<T\left(\partial B_{r}\right),\right.\right. \\
& \left.\left.e_{V}\left(T\left(\partial B_{A}\right)\right) f\left(X\left(T\left(\partial B_{A}\right)\right)\right)\right]\right\} \\
& =\int_{\partial B_{2 A}} K_{B_{2 A}}^{V}(z, u)\left[\int_{\partial B_{A}} K_{J_{A, r}}^{V}(u, w) f(w) \sigma(d w)\right] \sigma(d u) \\
& =\int_{\partial B_{A}}\left[\int_{\partial B_{2 A}} K_{B_{2 A}}^{V}(z, u) K_{J_{A, r}}^{V}(u, w) \sigma(d u)\right] f(w) \sigma(d w) .
\end{aligned}
$$


This proves (39). $\quad K_{B_{2 A}}^{V}(\cdot, \cdot)$ and $K_{J_{A, r}}^{V}(\cdot, \cdot) \quad(r<\infty)$ are continuous by Proposition 5. The continuity of $K_{J_{A}, \infty}^{V}=K_{B_{A}^{*}}^{V}$ is given by Lemma 5. So the kernel $\Phi_{r}$ given in (39) is continuous.

Lemma 7. $\sup _{(z, w) \in \partial B_{A} \times \partial B_{A}}\left|\Phi_{r}(z, w)-\Phi_{\infty}(z, w)\right| \rightarrow 0$ as $r \uparrow \infty$.

Proof. For $r \in(2 A, \infty)$, by the strong Markov property, we have for all $u \in$ $\partial B_{2 A}$, and $w \in \partial B_{A}$,

$$
K_{B_{A}^{*}}^{V}(u, w)=K_{J_{A, r}}^{V}(u, w)+\int_{\partial B_{r}} K_{J_{A, r}}^{V}(u, y) K_{B_{A}^{*}}^{V}(y, w) \sigma(d y) .
$$

By the estimate (33) for $K_{B_{A}^{*}}^{V}$ in Lemma 5,

$$
\left|K_{B_{A}^{*}}^{V}(u, w)-K_{J_{A, r}}^{V}(u, w)\right| \leq 2(d-2) C_{d} \frac{r^{2}-A^{2}}{A(r-A)^{d}} \int_{\partial B_{r}} K_{J_{A, r}}^{V}(u, y) \sigma(d y) .
$$

By Lemma 4,

$$
\begin{aligned}
\int_{\partial B_{r}} K_{J_{A, r}}^{V}(u, y) \sigma(d y) & =E^{u}\left[T\left(\partial B_{r}\right)<T\left(\partial B_{A}\right) ; e_{V}\left(T\left(\partial B_{r}\right)\right)\right] \\
& \leq E^{u}\left[\exp \left(\int_{0}^{\tau_{B_{A}^{*}}}\left|V\left(X_{s}\right)\right| d s\right)\right] \leq 2 .
\end{aligned}
$$

Then by (39)-(42) we obtain

$$
\left|\Phi_{r}(z, w)-\Phi_{\infty}(z, w)\right| \leq 4(d-2) c_{d} \frac{r^{2}-A^{2}}{A(r-A)^{d}} \int_{\partial B_{2 A}} K_{B_{2 A}}^{V}(z, w) \sigma(d u) .
$$

Since $\int_{\partial B_{2 A}} K_{B_{2 A}}^{V}(z, u) \sigma(d u)=E^{z}\left[e_{V}\left(\tau_{B_{2 A}}\right)\right]$, the lemma follows from (43) and the assumption (28).

We need some elementary properties for the integral operators in the Banach space $C(U)$, where $U$ is any compact set with a finite measure $\varphi$ on it. For any continuous function $Q(\cdot, \cdot)>0$ on $U \times U$ we define an operator in $C(U)$ :

$$
M_{Q} f(\cdot)=\int_{U} Q(\cdot, y) f(y) \mu(d y),
$$

and put $\lambda_{Q}=\lim _{n \rightarrow \infty} \sqrt[n]{\left\|\left(M_{Q}\right)^{n}\right\|}>0$.

Lemma 8. If $Q_{1}>Q_{2}>0$ on $U \times U$, then $\lambda_{Q_{1}}>\lambda_{Q_{2}}$.

Proof. Since $U \times U$ is compact, there exists an $\varepsilon>0$ such that $Q_{1}>(1+\varepsilon) Q_{2}$ on $U \times U$. Obviously, $\lambda_{Q}$ is nondecreasing with $Q$. Thus we have $\lambda_{Q_{1}} \geq$ $\lambda_{(1+\varepsilon) Q_{2}}=(1+\varepsilon) \lambda_{Q_{2}}>\lambda_{Q_{2}}$.

Lemma 9. If $\sup _{(x, y) \in U \times U}\left|Q_{n}(x, y)-Q(x, y)\right| \rightarrow 0$ as $n \rightarrow \infty$, then $\lambda_{Q_{n}} \rightarrow \lambda_{Q}$ as $n \rightarrow \infty$.

Proof. Since $Q>0$ in $U \times U$, for any $\varepsilon>0$, there exists $N \geq 1$ such that for all $n \geq N, x, y \in U,(1-\varepsilon) Q(x, y) \leq Q_{n}(x, y) \leq(1+\varepsilon) Q(x, y)$. Then $(1-\varepsilon) \lambda_{Q}=\lambda_{(1-\varepsilon) Q} \leq \lambda_{Q_{n}} \leq \lambda_{(1+\varepsilon) Q}=(1+\varepsilon) \lambda_{Q}$.

Now for the compact set $\partial B_{A}, r \in(2 A, \infty]$. Put

$$
\lambda_{r}(V)=\lim _{n \rightarrow \infty} \sqrt[n]{\left\|\left(S_{V, r}\right)^{n}\right\|},
$$


where $S_{V, r}$ is defined by (37) and is an integral operator in $C\left(\partial B_{A}\right)$ with a strictly positive and continuous kernel $\Phi_{r}$ by Lemma 6 .

The following lemma is a key step for the main theorem.

Lemma 10. For $r \in(2 A, \infty], E^{x}\left[e_{V}\left(\tau_{B_{r}}\right)\right] \not \equiv \infty$ if and only if $\lambda_{r}(V)<1$.

Proof. Put $f_{r}(z)=E^{z}\left[X\left(\sigma_{r}\right) \in \partial B_{r} ; e_{V}\left(\sigma_{r}\right)\right]$, if $r<\infty$, and

$$
f_{\infty}(z)=E^{z}\left[\sigma_{r}=\infty ; e_{V}\left(\sigma_{r}\right)\right] .
$$

Then for $z \in \partial B_{A}$, by the strong Markov property and the transient property of $\left\{X_{t}\right\}$ in $R^{d}(d \geq 3)$ for the case $r=\infty$, we have for each $r \in(2 A, \infty]$,

$$
E^{z}\left[e_{V}\left(\tau_{B_{r}}\right)\right]=\sum_{n=0}^{\infty}\left(S_{V, r}\right)^{n} f_{r}(z) .
$$

If $\lambda_{r}(V)<1$, then by (45), the series in (46) converges uniformly on $\partial B_{A}$, we have $E^{z}\left[e_{V}\left(\tau_{B_{r}}\right)\right]<\infty$. Conversely, if $E^{x}\left[e_{V}\left(\tau_{B_{r}}\right)\right] \not \equiv \infty$, then by Theorem 7 in [Z1] when $r<\infty$ and by Theorem 2 when $r=\infty, x \rightarrow E^{x}\left[e_{V}\left(\tau_{B_{r}}\right)\right]$ is a bounded continuous function in $\partial B_{A}$. Then by Dini's theorem, the convergence in the series in (46) is uniform on $\partial B_{A}$. Since $f_{r}(z)$ is a strictly positive and continuous function on $\partial B_{A}, c=\min _{z \in \partial B_{A}} f_{r}(z)>0$. By the uniform convergence we can find an integer $N$ such that $\left\|\left(S_{V, r}\right)^{N} f_{r}\right\|<c$. Therefore $\left\|\left(S_{V, r}\right)^{N}\right\|=\left\|\left(S_{V, r}\right)^{N} 1\right\| \leq c^{-1}\left\|\left(S_{V, r}\right)^{N} f_{r}\right\|<1$, and then $\sqrt[N]{\left\|\left(S_{V, r}\right)^{N}\right\|}<1$. Thus we obtain $\lambda_{V}(V)=\inf _{n} \sqrt[n]{\left\|\left(S_{V, r}\right)^{n}\right\|}<1$.

\section{Lemma 11.}

$$
\lim _{r \rightarrow \infty} \lambda_{r}(V)=\lambda_{\infty}(V) .
$$

Proof. (47) follows from Lemma 7 and Lemma 9.

Lemma 12. For each $r \in(2 A, \infty)$,

$$
\lambda_{r}(V)<\lambda_{\infty}(V) .
$$

Proof. Noticing $B_{A}^{*}=J_{A, \infty}$ we have by (39) and (40), for $2 A<r<\infty$ and any $z, w \in \partial B_{A}$,

$$
\begin{aligned}
\Phi_{\infty}(z, w)-\Phi_{r}(z, w) \\
\quad=\int_{\partial B_{2 A}} \int_{\partial B_{r}} K_{B_{2 A}}^{V}(z, u) K_{J_{A, r}}^{V}(u, y) K_{B_{A}^{*}}^{V}(y, w) \sigma(d u) \sigma(d y)>0 .
\end{aligned}
$$

Thus (48) follows from Lemma 8.

Lemma 13. $H \equiv-\Delta / 2+V \geq 0$ if and only if $\lambda_{\infty}(V) \leq 1$.

Proof. By Theorem 1 and Lemma 10, we have that $H \geq 0$ if and only if $\lambda_{r}(V)<1$ for $2 A<r<\infty$. By Lemma 11 and Lemma 12, the latter condition is equivalent to $\lambda_{\infty}(V) \leq 1$.

Lemma 14. Suppose $\left\{V_{n}\right\} \subseteq K_{d}^{\infty}$ and $\lim _{n \rightarrow \infty}\left\|V_{n}-V\right\| \|=0$. Then for sufficiently large $n, V_{n}$ satisfies the condition (26)-(28) and

$$
\lim _{n \rightarrow \infty} \lambda_{\infty}\left(V_{n}\right)=\lambda_{\infty}(V) .
$$


Proof. Obviously, (26) and (27) hold for $V_{n}$ when $n$ is sufficiently large. Since (28) holds for $V$, by Theorem 4 in [Z4] and Lemma A.4.3 in [A-S], there is $\beta>1$ such that

$$
\sup _{x \in B_{2 A}} E^{x}\left[e_{\beta V}\left(\tau_{B_{2 A}}\right)\right]<\infty .
$$

By Lemma 1 , it is easy to see that for sufficiently large $n$,

$$
\sup _{x \in B_{2 A}} E^{x}\left[e_{\beta\left(V_{n}-V\right) /(\beta-1)}\left(\tau_{B_{2 A}}\right)\right]<\infty .
$$

Thus, for $x \in B_{2 A}$, by Hölder's inequality, (50) and (51)

$$
\begin{aligned}
E^{x}\left[e_{V n}\left(\tau_{B_{2 A}}\right)\right] & =E^{x}\left[e_{V}\left(\tau_{B_{2 A}}\right) e_{V_{n}-V}\left(\tau_{B_{2 A}}\right)\right] \\
& \leq\left\{E^{x}\left[e_{\beta V}\left(\tau_{B_{2 A}}\right)\right]\right\}^{1 / \beta}\left\{E^{x}\left[e_{\beta\left(V_{n}-V\right) /(\beta-1)}\left(\tau_{B_{2 A}}\right)\right]\right\}^{(\beta-1) / \beta}<\infty,
\end{aligned}
$$

i.e., (28) holds for $V_{n}$.

In order to prove (49), let $\Phi_{\infty}^{n}(z, w)=\int_{\partial B_{2 A}} K_{B_{2 A}}^{V_{n}}(z, u) K_{B_{A}^{*}}^{V_{n}}(u, w) \sigma(d u)$. By Lemma 9 , we need only prove that

$$
\sup _{(z, w) \in \partial B_{A} \times \partial B_{A}}\left|\Phi_{\infty}^{n}(z, w)-\Phi_{\infty}(z, w)\right| \rightarrow 0 \quad \text { as } n \rightarrow \infty .
$$

By (39), it suffices to prove that

$$
\sup _{(z, u) \in \partial B_{A} \times \partial B_{2 A}}\left|K_{B_{2 A}}^{V_{n}}(z, u)-K_{B_{2 A}}^{V}(z, u)\right| \rightarrow 0,
$$

and

$$
\sup _{(u, w) \in \partial B_{2 A} \times \partial B_{A}}\left|K_{B_{A}^{*}}^{V_{n}}(u, w)-K_{B_{A}^{*}}^{V}(u, w)\right| \rightarrow 0,
$$

as $n \rightarrow \infty$. By (50) and the conditioned gauge theorem (see Theorem 1 in $[\mathrm{Z2}])$, we have

$$
M \equiv \sup _{(z, u) \in B_{2 A} \times a B_{2 A}} E_{u, B_{2 A}}^{z}\left[e_{\beta V}\left(\tau_{B_{2 A}}\right)\right]<\infty .
$$

Take an integer $N \geq \beta /(\beta-1)$, then by Hölder's inequality,

$$
\begin{aligned}
& \left|E_{u, B_{2 A}}^{z}\left[e_{V_{n}}\left(\tau_{B_{2 A}}\right)\right]-E_{u, B_{2 A}}^{z}\left[e_{V}\left(\tau_{B_{2 A}}\right)\right]\right| \\
& \quad=\left|E_{u, B_{2 A}}^{z}\left[e_{V}\left(\tau_{B_{2 A} A}\right)\left(e_{V_{n}-V}\left(\tau_{B_{2 A}}\right)-1\right)\right]\right| \\
& \quad \leq M^{1 / \beta}\left\{E_{u, B_{2 A}}^{z}\left[\exp \int_{0}^{\tau_{B_{2 A}}}\left|\left(V_{n}-V\right)\left(X_{s}\right)\right| d s-1\right]^{N}\right\}^{1 / N} .
\end{aligned}
$$

By a simple inequality: for $N \geq 1$ and $x>0$.

$$
\left(e^{x}-1\right)^{N} \leq \sum_{k=0}^{N}\left(\begin{array}{l}
N \\
k
\end{array}\right)\left(e^{k x}-1\right),
$$

and Lemma 3,

$$
\begin{aligned}
E_{u, B_{2 A} A}^{z} & {\left[\exp \int_{0}^{\tau_{B_{2 A}}}\left|\left(V_{n}-V\right)\left(X_{s}\right)\right| d s-1\right]^{N} } \\
\leq & \sum_{k=0}^{N}\left(\begin{array}{l}
N \\
k
\end{array}\right)\left[\frac{1}{1-2 k C(d)\left|\| V_{n}-V\right| \mid}-1\right] .
\end{aligned}
$$


For $(z, u) \in \partial B_{A} \times \partial B_{2 A}$, by (13),

$$
K_{B_{2 A}}(z, u)=(d-2) c_{d} \frac{4 A^{2}-|z|^{2}}{2 A|z-u|^{d}} \leq 2(d-2) c_{d} A^{1-d} .
$$

Let $W$ be one of $V$ and $V_{n}, n \geq 1$, we have by Corollary 2 in [Z3],

$$
K_{B_{2 A}}^{W}(z, u)=K_{B_{2 A}}(z, u) E_{z, B_{2 A}}^{x}\left[e_{W}\left(\tau_{B_{2 A}}\right)\right] \text {. }
$$

Since $\left\|V-V_{n}\right\| \rightarrow 0$ as $n \rightarrow \infty$, (53) follows from (55)-(58).

(54) follows in a similar and easier way.

Lemma 15. If $V_{1} \leq V_{2}$ and $V_{1} \not \equiv V_{2}$ a.e. in $R^{d}$, then

$$
\lambda_{\infty}\left(V_{1}\right)>\lambda_{\infty}\left(V_{2}\right) \text {. }
$$

Proof. Let

$$
\Phi_{\infty}^{i}(z, w)=\int_{\partial B_{2 A}} K_{B_{2 A}}^{V_{i}}(z, u) K_{B_{A}^{*}}^{V_{i}}(u, w) \sigma(d u) \quad(i=1,2) .
$$

By Lemma 8, we need only prove that for each $(z, w) \in \partial B_{A} \times \partial B_{A}$,

$$
\Phi_{\infty}^{1}(z, w)>\Phi_{\infty}^{2}(z, w) .
$$

Since $V_{1} \leq V_{2}$, we have by (35) and (58),

$$
K_{B_{2 A}}^{V_{1}}(z, u) \geq K_{B_{2 A}}^{V_{2}}(z, u) \text { for each }(z, u) \in \partial B_{A} \times \partial B_{2 A},
$$

and

$$
K_{B_{A}^{*}}^{V_{1}}(u, w) \geq K_{B_{A}^{*}}^{V_{2}}(u, w) \text { for each }(u, w) \in \partial B_{2 A} \times \partial B_{A} .
$$

Since $V_{1} \not \equiv V_{2}$ a.e. in $R^{d}$, we have either $V_{1} \not \equiv V_{2}$ a.e. in $B_{2 A}$ or $V_{1} \not \equiv V_{2}$ a.e. in $B_{A^{*}}$. In the former case

$$
\begin{aligned}
E_{u, B_{2 A}}^{z} & {\left[-\int_{0}^{\tau_{B_{2 A}}} V_{1}\left(X_{t}\right) d t+\int_{0}^{\tau_{B_{2 A}}} V_{2}\left(X_{t}\right) d t\right] } \\
= & \int_{B_{2 A}} \frac{G_{B_{2 A}}(z, y)\left[V_{2}(y)-V_{1}(y)\right] K_{B_{2 A}}(y, u)}{K_{B_{2 A}}(z, u)} d y>0 .
\end{aligned}
$$

Therefore by $V_{1} \leq V_{2}$ in $R^{d}$ we have

$$
E_{u, B_{2 A}}^{z}\left[e_{V_{1}}\left(\tau_{B_{2 A}}\right)\right]>E_{u, B_{2 A}}^{z}\left[e_{V_{2}}\left(\tau_{B_{2 A}}\right)\right] \text {. }
$$

This together with (58) shows that

$$
K_{B_{2 A}}^{V_{1}}(z, u)>K_{B_{2 A}}^{V_{2}}(z, u) .
$$

So (61) follows from (60), (63), and (65). Similarly in the latter case, we have

$$
K_{B_{A}^{*}}^{V_{1}}(u, w)>K_{B_{A}^{*}}^{V_{2}}(u ; w) .
$$

Then (61) follows from (60), (62), and (66).

Lemma 16. For all $z \in \partial B_{4 A}$ and $y \in B_{6 A}^{*}$, we have

$$
e^{-1} \leq E^{z}\left[\tau_{B_{A}^{*}}=\infty ; e_{V}\left(\tau_{B_{A}^{*}}\right)\right]
$$

and

$$
e^{-8} \leq E_{y}^{z}\left[\xi<\tau_{B_{A}^{*}} ; e_{V}(\xi)\right]
$$


(Note that $E_{y}^{z}$ denotes $E_{y, R^{d}}^{z}$ and $\xi$ is the lifetime of the conditional $y$ process.)

Proof. For $z \in \partial B_{4 A}$,

$$
P^{z}\left(\tau_{B_{A}^{*}}=\infty\right)=1-\frac{A^{d-2}}{|z|^{d-2}}=1-4^{2-d} \geq \frac{3}{4},
$$

and by (26),

$$
E^{z}\left[\int_{0}^{\tau_{B_{A}^{*}}}\left|V\left(X_{s}\right)\right| d s\right] \leq c_{d} \int_{|y|>A} \frac{|V(y)|}{|z-y|^{d-2}} d y \leq 2^{-5} .
$$

Then (67) follows from Lemma 2 and a simple inequality: $\frac{3}{4} e^{-4 / 3} 2^{-5} \geq e^{-1}$.

Now for $z \in \partial B_{4 A}$ and $y \in B_{6 A}^{*}$, by (13) with $D_{A}=B_{A}^{*}$,

$$
\begin{aligned}
P_{y}^{z}\left(\tau_{B_{A}^{*}}<\xi\right) & =|z-y|^{d-2} E^{z}\left(\tau_{B_{A}^{*}}<\infty ;\left|X\left(\tau_{B_{A}^{*}}\right)-y\right|^{2-d}\right) \\
& =(d-2) c_{d}|z-y|^{d-2} \int_{\partial B_{A}} \frac{|z|^{2}-A^{2}}{A|z-w|^{d}|w-y|^{d-2}} \sigma(d w) \\
& =15(d-2) c_{d} A \int_{\partial B_{A}} \frac{1}{|z-w|^{2}}\left(\frac{|z-y|}{|z-w||w-y|}\right)^{d-2} \sigma(d w) \\
& \leq 15(d-2) c_{d} A \int_{\partial B_{A}} \frac{1}{(3 A)^{2}}\left(|z-w|^{-1}+|w-y|^{-1}\right)^{d-2} \sigma(d w) \\
& \leq \frac{5}{3}(d-2) c_{d} A^{-1} \int_{\partial B_{A}}\left(\frac{1}{3 A}+\frac{1}{5 A}\right)^{d-2} \sigma(d w) \\
& =\frac{5}{3}\left(\frac{8}{15}\right)^{d-2} \leq \frac{8}{9} .
\end{aligned}
$$

So $P_{y}^{z}\left(\xi<\tau_{B_{A}^{*}}\right) \geq 1-\frac{8}{9}=\frac{1}{9}$ and by $(26)$,

$$
\begin{aligned}
E_{y}^{z}\left[\int_{0}^{\tau_{B_{A}^{*}}}\left|V\left(X_{s}\right)\right| d s\right] & \leq c_{d} \int_{|w| \geq A} \frac{|z-y|^{d-2}|V(w)|}{|z-w|^{d-2}|w-y|^{d-2}} d w \\
& \leq 2^{d-2} c_{d} \sup _{|x| \geq A} \int_{|w| \geq A} \frac{|V(w)|}{|x-w|^{d-2}} d w<\frac{1}{2} .
\end{aligned}
$$

Thus (68) follows from Lemma 2 and a simple inequality $\frac{1}{9} e^{-9 / 2} \geq e^{-8}$.

By similar arguments as those in Lemma 16, we have

Lemma 17. Let $0<r \leq a$, where $a$ is given in (27). Then for any ball $B=$ $B(w, r) \quad\left(w \in R^{d}\right), z \in \partial B(w, r / 5)$ and $y \in B(w, r / 10)$, we have

$$
e^{-3} \leq E_{y}^{z}\left[\xi=\tau_{B} ; e_{V}\left(\tau_{B}\right)\right]
$$

For $V \in K_{d}^{\infty}$, put

$$
u_{0}(x, y)=E_{y}^{x}\left[e_{V}(\xi)\right] \equiv E_{y}^{x}\left[\exp \left(-\int_{0}^{\xi} V\left(X_{s}\right) d s\right)\right], \quad x \neq y,
$$

where $\xi$ is the life time of the $y$-process. Note $E_{y}^{x}$ denotes $E_{y, R^{d}}^{x}$. 
Lemma 18. There exists a function $Q$ on $\overline{B_{4 A}}$ with $0<Q \leq \infty$ such that for each $x \in \overline{B_{4 A}}$,

$$
e^{-1} Q(x) \leq u_{0}(x) \leq 2 Q(x),
$$

and for each $x \in \overline{B_{4 A}}, y \in B_{6 A}^{*}$,

$$
e^{-8} 5^{2-d} Q(x) \leq u_{0}(x, y) \leq 5^{d-1} Q(x) .
$$

Proof. For $x \in \overline{B_{4 A}}$ and $y \in B_{6 A}^{*}$, define a sequence of stopping times under $P^{x}$ and $P_{y}^{x}$, respectively, $T_{0}=0$, for $n \geq 1$ :

$$
\begin{aligned}
& T_{2 n-1}=T_{2 n-2}+\tau_{B_{44}} \circ \theta_{T_{2 n-2}} \text { on } \begin{cases}\left(T_{2 n-2}<\infty\right) & \text { under } P^{x}, \\
\left(T_{2 n-2}<\xi\right) & \text { under } P_{y}^{x} .\end{cases} \\
& T_{2 n}=T_{2 n-1}+\tau_{B_{A}^{*}} \circ \theta_{T_{2 n-1}} .
\end{aligned}
$$

Put

$$
Q(x)=\sum_{n=1}^{\infty} E^{x}\left[T_{2 n-2}<\infty ; e_{V}\left(T_{2 n-1}\right)\right] .
$$

By the strong Markov property, we have for $x \in \overline{B_{4 A}}$,

$$
\begin{aligned}
u_{0}(x) & =\sum_{n=1}^{\infty} E^{x}\left[T_{2 n-2}<\infty \text { and } T_{2 n}=\infty ; e_{V}(\infty)\right] \\
& =\sum_{n=1}^{\infty} E^{x}\left\{T_{2 n-2}<\infty ; e_{V}\left(T_{2 n-1}\right) E^{X\left(T_{2 n-1}\right)}\left[\tau_{B_{A}^{*}}=\infty, e_{V}\left(\tau_{B_{A}^{*}}\right)\right]\right\}
\end{aligned}
$$

Then by Lemma 4 and Lemma 16 (67) we get (71) from (73).

Now for $x \in \overline{B_{4 A}}$ and $y \in B_{6 A}^{*}$, by the strong Markov property of the $y$-process, we have

$$
u_{0}(x, y)=\sum_{n=1}^{\infty} E_{y}^{x}\left\{T_{2 n-2}<\xi ; e_{V}\left(T_{2 n-1}\right) E_{y}^{X\left(T_{2 n-1}\right)}\left[\xi<\tau_{B_{A}^{*}} ; e_{V}(\xi)\right]\right\} .
$$

For each $n \geq 1, X\left(T_{2 n-1}\right) \in \partial B_{4 A}$, on $\left(T_{2 n-2}<\xi\right)$, then by Lemma $16(68)$, Lemma 3 and (26), we obtain

$$
\begin{aligned}
e^{-8} & \leq E_{y}^{X\left(T_{2 n-1}\right)}\left[\xi<\tau_{B_{A}^{*}} ; e_{V}(\xi)\right] \\
& \leq E_{y}^{X\left(T_{2 n-1}\right)}\left[\exp \left(\int_{0}^{\tau_{B_{A}^{*}}}\left|V\left(X_{s}\right)\right| d s\right)\right] \leq 2,
\end{aligned}
$$

and

$$
\begin{aligned}
& E_{y}^{x}\left\{T_{2 n-2}<\xi ; e_{V}\left(T_{2 n-1}\right)\right\} \\
& \quad=|x-y|^{d-2} E^{x}\left\{T_{2 n-2}<\infty ;\left|X\left(T_{2 n-1}\right)-y\right|^{2-d} e_{V}\left(T_{2 n-1}\right)\right\} .
\end{aligned}
$$

For $z \in \partial B_{4 A}, x \in \bar{B}_{4 A}$, and $y \in B_{6 A}^{*}$,

$$
\frac{1}{5} \leq \frac{|x-y|}{|z-x|+|x-y|} \leq \frac{|x-y|}{|z-y|} \leq \frac{|x-z|}{|z-y|}+1 \leq 5 .
$$

Thus (72) follows from (74)-(77). 
Lemma 19. There exists a constant $c_{1}>0$ depending on $d, A$ and $a$ only such that for each $x \in \overline{B_{4 A}}, y$ and $y^{\prime} \in \overline{B_{8 A}}$ with $|y-x| \geq a$ and $\left|y^{\prime}-x\right| \geq a$,

$$
u_{0}(x, y) \leq c_{1} u_{0}\left(x, y^{\prime}\right) \text {. }
$$

Proof. Fix $x \in \overline{B_{4 A}}$. For any $y \in \bar{B}_{8 A}$ with $|y-x| \geq a$ and a ball $B=$ $B(w, a / 8)$ containing $y$. Set a sequence of stopping times:

$$
\begin{array}{ll}
S_{0}=0, \quad & S_{2 n-1}=S_{2 n-2}+\tau_{B^{*}(w, a / 4)} \circ \theta_{S_{2 n-2}}, \\
& S_{2 n}=S_{2 n-1}+\tau_{B(w, a)} \circ \theta_{S_{2 n-1}} \quad(n \geq 1) .
\end{array}
$$

Then

$$
\begin{aligned}
u_{0}(x, y) & =\sum_{n=1}^{\infty} E_{y}^{x}\left[\xi=S_{2 n} ; e_{V}(\xi)\right] \\
& =\sum_{n=1}^{\infty} E_{y}^{x}\left\{S_{2 n-2}<\xi ; e_{V}\left(S_{2 n-1}\right) E_{y}^{X\left(S_{2 n-1}\right)}\left[\xi=\tau_{B(w, a)} ; e_{V}(\xi)\right]\right\}
\end{aligned}
$$

By (24) in Lemma 3, condition (27) and Lemma 17, we have for each $n \geq 1$, $\left(X\left(S_{2 n-1}\right) \in \partial B(w, a / 4)\right)$,

$$
e^{-3} \leq E_{y}^{X\left(S_{2 n-1}\right)}\left[\xi=\tau_{B(w, a)} ; e_{V}\left(\tau_{B(w, a)}\right)\right] \leq 2,
$$

and

$$
\begin{aligned}
& E_{y}^{x}\left\{S_{2 n-2}<\xi ; e_{V}\left(S_{2 n-1}\right)\right\} \\
& \quad=|x-y|^{d-2} E^{x}\left\{S_{2 n-2}<\infty ;\left|X\left(S_{2 n-1}\right)-y\right|^{2-d} e_{V}\left(S_{2 n-1}\right)\right\} .
\end{aligned}
$$

For each $z \in \partial B(w, a / 4)$, we have

$$
1 \leq \frac{a}{a / 4+a / 8} \leq \frac{|x-y|}{|z-y|} \leq \frac{12 A}{a / 8}=96 \frac{A}{a}
$$

Thus it follows from (79) and (80) that

$$
\begin{gathered}
e^{-3} \sum_{n=1}^{\infty} E_{y}^{x}\left\{S_{2 n-2}<\xi ; e_{V}\left(S_{2 n-1}\right)\right\} \leq u_{0}(x, y) \\
\quad \leq 2 \sum_{n=1}^{\infty} E_{y}^{x}\left\{S_{2 n-2}<\xi ; e_{V}\left(S_{2 n-1}\right)\right\}
\end{gathered}
$$

and it follows from $(81)-(83)$ that

$$
\begin{aligned}
& e^{-3} \sum_{n=1}^{\infty} E^{x}\left\{S_{2 n-2}<\xi ; e_{V}\left(S_{2 n-1}\right)\right\} \leq u_{0}(x, y) \\
& \quad \leq 2\left(\frac{96 A}{a}\right)^{d-2} \sum_{n=1}^{\infty} E^{x}\left\{S_{2 n-2}<\xi ; e_{V}\left(S_{2 n-1}\right)\right\} .
\end{aligned}
$$

By (84), for any $y$ and $y^{\prime}$ in $\overline{B_{8 A}}$ with $|y-x| \geq a$ and $\left|y^{\prime}-x\right| \geq a$, if $y$ and $y^{\prime}$ are in the same ball $B(w, a / 8)$, we have

$$
u_{0}(x, y) \leq 2 e^{d}(96 A / a)^{d-2} u_{0}\left(x, y^{\prime}\right) .
$$

Thus (78) follows from (85) and a ball chain argument. 
Lemma 20. For any $B=B(0, R), 0<R<\infty$, if $G_{B}^{V}(x, y)>0$ exists for $x, y \in B, x \neq y$, then we have

$$
E_{y}^{x}\left[\xi<\tau_{B} ; e_{V}(\xi)\right] \leq G_{B}^{V}(x, y) / G(x, y) .
$$

Proof. By [Z3, Theorem 7], the existence of $G_{B}^{V}$ implies that

$$
E_{y, B}^{x}\left[e_{V}(\xi)\right]=G_{B}^{V}(x, y) / G_{B}(x, y),
$$

and there is $c>0$ such that

$$
\inf _{x, y \in B} E_{y, B}^{x}\left[e_{V}(\xi)\right] \geq c .
$$

Fix $x$ and $y$ in $B, x \neq y$. Then for any $0<r<\min (|x-y|, R-|x|, R-$ $|y|)$, put $T_{r}=$ hitting time on $\partial B(y, r)$. We have $T_{r} \uparrow \xi$, a.s. as $r \downarrow 0$. For each such $r$, by (88),

$$
E_{y, B}^{x}\left[e_{V}(\xi) \mid \mathscr{F}_{T_{r}}\right]=e_{V}\left(T_{r}\right) E_{y, B}^{X\left(T_{r}\right)}\left[e_{V}(\xi)\right] \geq c e_{V}\left(T_{r}\right) .
$$

Hence $\left\{e_{V}\left(T_{r}\right)\right\}$ are uniformly integrable as $r \downarrow 0$, then we have

$$
\lim _{r \downarrow 0} E_{y, B}^{x}\left[e_{V}\left(T_{r}\right)\right]=E_{y, B}^{x}\left[e_{V}(\xi)\right] .
$$

For each small $r$, we have

$$
E_{y, B}^{x}\left[e_{V}\left(T_{r}\right)\right]=\frac{1}{G_{B}(x, y)} E^{x}\left[T_{r}<\tau_{B} ; G_{B}\left(X\left(T_{r}\right), y\right) e_{V}\left(T_{r}\right)\right] .
$$

Since $\left|X\left(T_{r}\right)-y\right|=r$,

i.e.,

$$
\frac{c_{d}}{r^{d-2}}-\frac{c_{d}}{(R-|y|)^{d-2}} \leq G_{B}\left(X\left(T_{r}\right), y\right) \leq \frac{c_{d}}{r^{d-2}},
$$

$$
1-\frac{r^{d-2}}{(R-|y|)^{d-2}} \leq \frac{G_{B}\left(X\left(T_{r}\right), y\right)}{G\left(X\left(T_{r}\right), y\right)} \leq 1 .
$$

By (89)-(91), we have

$$
\frac{1}{G_{B}(x, y)} \lim _{r \downarrow 0} E^{x}\left[T_{r}<\tau_{B} ; G\left(X\left(T_{r}\right), y\right) e_{V}\left(T_{r}\right)\right]=E_{y, B}^{x}\left[e_{V}(\xi)\right] .
$$

This is equivalent to

$$
\frac{G(x, y)}{G_{B}(x, y)} \lim _{r \downarrow 0} E_{y}^{x}\left[T_{r}<\tau_{B} ; e_{V}\left(T_{r}\right)\right]=E_{y, B}^{x}\left[e_{V}(\xi)\right] .
$$

By Fatou's lemma and (87), we obtain

This is the inequality (86).

$$
\frac{G(x, y)}{G_{B}(x, y)} E_{y}^{x}\left[\xi<\tau_{B} ; e_{V}(\xi)\right] \leq \frac{G_{B}^{V}(x, y)}{G_{B}(x, y)} .
$$

\section{Main Result}

Let $V \in K_{d}^{\infty} \quad(d \geq 3)$ and put

$$
\begin{aligned}
H & =-\frac{\Delta}{2}+V, \\
u_{0}(x) & =E^{x}\left[e_{V}(\infty)\right] \equiv E^{x}\left[\exp \left(-\int_{0}^{\infty} V\left(X_{s}\right) d s\right)\right],
\end{aligned}
$$

and 


$$
u_{0}(x, y)=E_{y}^{x}\left[e_{V}(\xi)\right] \equiv E_{y}^{x}\left[\exp \left(-\int_{0}^{\xi} V\left(X_{s}\right) d s\right)\right], \quad x \neq y
$$

We choose $A>0$ and $a>0$ satisfying (26) and (27), respectively, and define the "shuttle operator": $S_{V}=S_{V, \infty}$, as in (37) $(r=\infty)$ and its spectral radius $\lambda(V)=\lambda_{\infty}(V)$, as in (45) $(r=\infty)$.

Main Theorem. Let $V \in K_{d}^{\infty}(d \geq 3)$. Then the following conditions are equivalent:

(a) There exists $\varepsilon>0$ such that for any $q \in K_{d}^{\infty}$ with $\|q\| \mid<\varepsilon, H+q \geq 0$.

(b) There exists $\beta>0$ such that $-\Delta / 2+(1+\beta) V \geq 0$.

(c) There exists a positive Green function $G^{V}(\cdot, \cdot)$ for $H$.

(d) There exists a solution $u$ of $H u=0$ with $\inf _{x} u(x)>0$.

(e) $u_{0}(x) \not \equiv \infty$ in $R^{d}$.

(f) $u_{0}(x, y)$ is bounded in $R^{d} \times R^{d} \backslash\left\{(x, x): x \in R^{d}\right\}$.

(g) $\lambda(V)<1$.

Remark 1. We have other equivalent conditions in terms of small perturbations:

(a1) For any bounded Borel function $q$ with compact support, there exists $\varepsilon>0$ such that $H+\varepsilon q \geq 0$.

(a2) There exists a bounded Borel function $q$ with compact support, $q \leq 0$ and $q \not \equiv 0$ a.e. in $R^{d}$ such that $H+q \geq 0$.

(a3) There exists a function $q \in K_{d}^{\infty}, q \leq 0$, and $q \not \equiv 0$ a.e. in $R^{d}$ such that $H+q \geq 0$.

and in terms of positive solutions:

(d1) There exists a continuous solution $u>0$ of $H u=0$ with the limit: $\lim _{|x| \rightarrow \infty} u(x)>0$.

(d2) There exists a solution $u$ of $H u=0$ with $0<\inf _{x} u(x) \leq \sup _{x} u(x)<$ $\infty$.

Remark 2. By Theorem 2 (generalized "Gauge Theorem"), condition (e) is equivalent to

(e1) $u_{0}(x)$ is bounded in $R^{d}$.

Remark 3. We shall prove that condition (f) is equivalent to

(f1) $u_{0}\left(x_{0}, y_{0}\right)<\infty$ for some $\left(x_{0}, y_{0}\right)$ in $R^{d} \times R^{d}, x_{0} \neq y_{0}$.

This equivalence can be regarded as a generalized conditioned Gauge Theorem (see $[\mathrm{C}-\mathrm{F}-\mathrm{Z}]$ and $[\mathrm{F}]$ ).

Proof of Main Theorem. Since every bounded Borel function with compact support is in $K_{d}^{\infty}$, we have obvious implications: (a) $\Rightarrow(\mathrm{a} 1) \Rightarrow(\mathrm{a} 2) \Rightarrow(\mathrm{a} 3)$.

(a3) $\Rightarrow(\mathrm{g})$ : Since $q \leq 0, H \geq H+q \geq 0$. By Theorem $1,(28)$ is satisfied for $V$ and $V+q$. By Lemma 13, $H+q \geq 0$ implies $\lambda(V+q) \leq 1$. Then we obtain by Lemma 15 that $\lambda(V)<\lambda(V+q) \leq 1$, i.e., (g) holds.

$(\mathrm{g}) \Rightarrow(\mathrm{a})$ : Since $\lambda(V)<1$, by Lemma 14 , there exists $\varepsilon>0$ such that if $\|q\|<\varepsilon$, then $\lambda(V+q)<1$. Therefore we have $H+q=-\Delta / 2+(V+q) \geq 0$ by Lemma 13. Thus (a) and (g) are equivalent.

It follows from Lemma 10 with $r=\infty$ that (e) and (g) are equivalent.

(e) $\Rightarrow(\mathrm{d} 1)$ : By the second statement of Theorem $2, u_{0}>0$ is a continuous 
solution of $H u=0$. By the equality (32) and Proposition 3(ii) we have

$$
\begin{aligned}
\lim _{|x| \rightarrow \infty} u_{0}(x) & =1-\lim _{|x| \rightarrow \infty} \int_{R^{d}} \frac{c_{d} V(y) u_{0}(y)}{|x-y|^{d-2}} d y \\
& =1-c_{d} \int_{R^{d}}\left[\lim _{|x| \rightarrow \infty} \frac{V(y) u_{0}(y)}{|x-y|^{d-2}}\right] d y \\
& =1 .
\end{aligned}
$$

So (d1) holds.

(d) $\Rightarrow$ (e): Suppose there is a solution $u$ of $H u=0$ with $u(x) \geq c>0$ for all $x \in R^{d}$. Then for any $R>0$, we have

$$
u(0)=E^{0}\left[e_{V}\left(\tau_{B(0, R)}\right) u\left(X\left(\tau_{B(0, R)}\right)\right)\right] \geq c E^{0}\left[e_{V}\left(\tau_{B(0, R)}\right)\right] .
$$

By Fatou's lemma, we obtain

$$
E^{0}\left[e_{V}(\infty)\right] \leq \lim _{R \rightarrow \infty} E^{0}\left[e_{V}\left(\tau_{B(0, R)}\right)\right] \leq c^{-1} u(0)<\infty,
$$

i.e., (e) holds.

Since we have (d1) $\Rightarrow(\mathrm{d} 2) \Rightarrow(\mathrm{d})$ obviously, we proved (d) and (e) are equivalent.

(e) $\Rightarrow$ (f): By Theorem 2, we may suppose $M \equiv \sup _{x \in R^{d}} u_{0}(x)<\infty$. Then by (71) and (72) in Lemma 18 we have that for $x \in \overline{B_{4 A}}$ and $y \in B_{6 A}^{*}$,

$$
u_{0}(x, y) \leq c_{0} M,
$$

where $c_{0}=e 5^{d-1}$. By (92) and (78) in Lemma 19 we have that for $x \in \overline{B_{4 A}}$ and any $y$ in $\overline{B_{6 A}}$ with $|y-x| \geq a$,

$$
u_{0}(x, y) \leq c_{0} c_{1} M \text {. }
$$

Now for $x \in \overline{B_{2 A}}$ and $0<|y-x|<a$, by Lemma 3 and condition (27), we have

$$
\begin{aligned}
u_{0}(x, y)= & E_{y}^{x}\left\{\xi=\tau_{B(y, a)} ; e_{V}(\xi)\right\} \\
& +E_{y}^{x}\left\{\tau_{B(y, a)}<\xi ; u_{0}\left(X\left(\tau_{B(y, a)}\right), y\right)\right\} \\
\leq & E_{y}^{x}\left\{\exp \left(\int_{0}^{\tau_{B(y, a)}}\left|V\left(X_{s}\right)\right| d s\right)\right]+c_{0} c_{1} M \\
\leq & 2+c_{0} c_{1} M,
\end{aligned}
$$

since $\left|X\left(\tau_{B(y, a)}\right)\right| \leq|x|+|x-y|+a \leq 2 A+2 a \leq 4 A$, the first inequality in (94) follows from (93).

Now for $x \in B_{2 A}^{*}$ and $y \neq x$,

$$
\begin{aligned}
u_{0}(x, y) & =E_{y}^{x}\left[\xi \leq \tau_{B_{2 A}^{*}} ; e_{V}(\xi)\right]+E_{y}^{x}\left[\tau_{B_{2 A}^{*}}<\xi ; u_{0}\left(X\left(\tau_{B_{2 A}^{*}}\right), y\right)\right] \\
& \leq E_{y}^{x}\left[\exp \left(\int_{0}^{\tau_{B_{A}^{*}}}\left|V\left(X_{s}\right)\right| d s\right)\right]+\left(2+c_{0} c_{1} M\right) \\
& \leq 4+c_{0} c_{1} M .
\end{aligned}
$$

Thus (f) follows from (93)-(95). 
(f) $\Rightarrow(\mathrm{c}):$ Let $W \equiv \sup _{x, y \in R^{d}, x \neq y} u_{0}(x, y)<\infty$. then we have

$$
\begin{aligned}
u_{0}(x, y)-1 & =E_{y}^{x}\left[\exp \left(-\int_{0}^{\xi} V\left(X_{s}\right) d s\right)-1\right] \\
& =-E_{y}^{x}\left[\int_{0}^{\xi} V\left(X_{t}\right) \exp \left(-\int_{t}^{\xi} V\left(X_{s}\right) d s\right) d t\right] \\
& =-E_{y}^{x}\left\{\int_{0}^{\xi} V\left(X_{t}\right) E_{y}^{X_{t}}\left[e_{V}(\xi)\right] d t\right\} \\
& =-E_{y}^{x}\left[\int_{0}^{\xi} V\left(X_{t}\right) u_{0}\left(X_{t}, y\right)\right] \\
& =-\int_{R^{d}} \frac{G(x, z) V(z) G(z, y)}{G(x, y)} u_{0}(z, y) d z
\end{aligned}
$$

The equalities in (96) can be justified by Fubini's Theorem and the inequalities

$$
\begin{aligned}
\int_{R^{d}} & \frac{G(x, z)|V(z)| G(z, y)}{G(x, y)} u_{0}(z, y) d z \\
& \leq W 2^{d-2}\left(c_{d} \int_{R^{d}} \frac{|V(z)|}{|x-z|^{d-2}} d z+c_{d} \int_{R^{d}} \frac{|V(z)|}{|y-z|^{d-2}} d z\right) \\
& \leq W c_{d} 2^{d-1}|| V \mid \|<\infty .
\end{aligned}
$$

Put $F(x, y)=u_{0}(x, y) G(x, y)>0, x \neq y$. Then by (96),

$$
F(x, y)=G(x, y)-\int_{R^{d}} G(x, z) V(z) F(z, y) d z .
$$

For $\psi \in C_{c}^{\infty}\left(R^{d}\right)$, put

$$
F \psi(\cdot) \equiv \int_{R^{d}} F(\cdot, y) \psi(y) d y, \quad G \psi(\cdot) \equiv \int_{R^{d}} G(\cdot, y) \psi(y) d y .
$$

By (97), $F \psi=G \psi-G[V(F \psi)]=G(\psi-V(F \psi))$. So we have $\Delta F \psi=$ $\Delta G[\psi-V(F \psi)]=-2[\psi-V(F \psi)]$, i.e., $(-\Delta / 2+V)(F \psi)=\psi$. This means that $F(\cdot, \cdot)$ is the positive Green function $G^{V}(\cdot, \cdot)$ for $H$. Hence (c) holds.

(c) $\Rightarrow(\mathrm{f} 1)$ : For any ball $B=B(0, R), R>0$, we have

$$
G_{B}^{V}(x, y) \leq G^{V}(x, y)<\infty, \quad x, y \in B .
$$

Then by Lemma 20 and (98), we have for any $x, y \in R^{d}$ with $x \neq y$ and $R>\max (|x|,|y|)$,

$$
E_{y}^{x}\left[\xi<\tau_{B(0, R)} ; e_{V}(\xi)\right] \leq \frac{G_{B}^{V}(x, y)}{G(x, y)} \leq \frac{G^{V}(x, y)}{G(x, y)} .
$$

Letting $R \uparrow \infty$, By Fatou's lemma, we obtain $E_{y}^{x}\left[e_{V}(\xi)\right] \leq G^{V}(x, y) / G(x, y)<$ $\infty$.

$(\mathrm{f} 1) \Rightarrow(\mathrm{e})$ : Suppose for some $x_{0}, y_{0}$ with $x_{0} \neq y_{0}, u_{0}\left(x_{0}, y_{0}\right)<\infty$. By enlarging $A$ and reducing $a$ (if necessary), we may assume that $x_{0} \in \overline{B_{4 A}}$, $y_{0} \in \overline{B_{8 A}}$, and $0<a \leq\left|x_{0}-y_{0}\right|$. Thus by Lemma 18 and Lemma 19 , we have $u_{0}\left(x_{0}\right)<\infty$, i.e., (e) holds. Thus we proved the equivalence among (e), (f), and (c). 
Since $(a) \Rightarrow(b)$, obviously, and we have proved $(g) \Rightarrow(a)$, our last implication is

(b) $\Rightarrow(\mathrm{g})$ : For each $t \in[0,1+\beta]$, put

$$
f(t) \equiv \ln [\lambda(t V)]=\lim _{n \rightarrow \infty} \frac{1}{n} \ln \left\|\left(S_{t V}\right)^{n}\right\| .
$$

Define a sequence of stopping times $\left\{T_{k}\right\}$ :

$$
T_{1}=\tau_{B_{2 A}}+\tau_{B_{A}^{*}} \circ \theta_{\tau_{B_{2 A}}},
$$

and

$$
T_{k+1}= \begin{cases}T_{k}+T_{1} \circ \theta_{T_{k}} & \text { if } T_{k}<\infty, \\ \infty & \text { if } T_{k}=\infty .\end{cases}
$$

Then for each $n \geq 1$,

$$
\left(S_{t V}\right)^{n} f(x)=E^{x}\left[T_{n}<\infty ; e_{t V}\left(T_{n}\right) f\left(X\left(T_{n}\right)\right)\right],
$$

and

$$
\left\|\left(S_{t V}\right)^{n}\right\|=\left\|\left(S_{t V}\right)^{n} 1\right\|=\sup _{x \in \partial B_{A}} E^{x}\left[T_{n}<\infty ; e_{t V}\left(T_{n}\right)\right] .
$$

Hence by the Cauchy-Schwarz inequality for any $t, s \in[0,1+\beta]$, we have

$$
\left\|S_{((t+s) / 2) V}^{n}\right\| \leq\left\|S_{t V}^{n}\right\|^{1 / 2}\left\|S_{s V}^{n}\right\|^{1 / 2} .
$$

Therefore the function $t \rightarrow \ln \left\|S_{t V}^{n}\right\|$ is convex, so is $f(t)$ by (99).

By Lemma $13, \lambda(t V) \leq 1, t \in[0,1+\beta]$, so $f(t) \leq 0$ on $[0,1+\beta]$. Since for $V \equiv 0, E^{x}\left[e_{0}(\infty)\right] \equiv 1$, by Lemma $10, \lambda(0)<1$, i.e., $f(0)<0$. We obtain that $f(1)<0$, since otherwise the convex function $f$ reaches its maximum at an interior point 1 in $[0,1+\beta], f$ must be identical to 0 on $[0,1+\beta]$, which contradicts $f(0)<0$. Thus we obtain that $\lambda(V)=\exp [f(1)]<1$.

\section{POSTSCRIPT}

The conjecture made in the Introduction was recently solved by V. Pinchover with an affirmative answer (a private communication).

\section{ACKNOWLEDGMENT}

I am grateful to F. Gesztesy for the valuable discussions and to the referee for the helpful suggestions and corrections.

\section{REFERENCES}

[A-S] M. Aizenman and B. Simon, Brownian motion and Harnack inequality for Schrödinger operators, Comm. Pure Appl. Math. 35 (1982), 209-273.

[A1] W. Allegretto, Criticality and the $\lambda$-property for elliptic equations, J. Differential Equations 69 (1987), 39-45.

[Ch] K. L. Chung, On stopped Feynman-Kac functional, Séminaire de Probabilités XIV (Univ. Strasbourg), Lecture Notes in Math., vol. 784, Springer-Verlag, Berlin, 1980.

[Ch-R] K. L. Chung and M. Rao, Feynman-Kac functional and Schrödinger equation, Sem. Stoch. Proc., Birkhäuser, Boston, Mass., 1981.

[Ch-V] K. L. Chung and S. R. S. Varadhan, Kac functional and Schrödinger equation, Studia Math. 68 (1980), 249-260. 
[C-F-Z] M. Cranston, E. Fabes, and Z. Zhao, Conditional gauge and potential theory for the Schrödinger operator, Trans. Amer. Math. Soc. 307 (1988), 171-194.

[D] J. L. Doob, Conditioned Brownian motion and the boundary limits of harmonic functions, Bull. Soc. Math. France 85 (1957), 431-458.

[F] N. Falkner, Feynman-Kac functionals and positive solutions of $\frac{1}{2} \Delta u+q u=0, \mathrm{Z}$. Wahrsch. Verw. Gebiete. 65 (1983), 19-31.

[H-Z] I. W. Herbst and Z. Zhao, Green's functions for the Schrödinger equation with short-range potentials, Duke Math. J. 59 (1989), 475-519.

[G-Z] F. Gesztesy and Z. Zhao, On critical and subcritical Sturm-Liouville operators, J. Funct. Anal. 98 (1991), 311-345.

[M1] M. Murata, Positive solutions and large time behaviours of Schrödinger semigroups, Simon's problem, J. Funct. Anal. 56 (1984), 300-310.

[M2] - Structure of positive solutions of $(-\Delta+V) u=0$ in $R^{n}$, Duke Math. J. 53 (1986), 869-943.

[Pi] Y. Pinchover, Criticality and ground states for second order elliptic equations, J. Differential Equations 80 (1989), 237-250.

[P-S] S. Port and C. Stone, Brownian motion and classical potential theory, Academic Press, New York, 1978.

[S1] B. Simon, Large time behavior of the $L^{p}$ norm of Schrödinger semigroups, J. Funct. Anal. 40 (1981), 66-83.

[S2] — Schrödinger semigroups, Bull. Amer. Math. Soc. (N.S.) 7 (1982), 447-526.

[Z1] Z. Zhao, Conditional gauge and unbounded potential, Z. Wahrsch. Verw. Gebiete. 65 (1983), 13-18.

[Z2] _ Uniform boundedness of conditional gauge and Schrödinger equations, Comm. Math. Phys. 93 (1984), 19-31.

[Z3] - Green function for Schrödinger operator and conditioned Feynman-Kac gauge, J. Math. Anal. Appl. 116 (1986), 309-334.

[Z4] _ An equivalence theorem for Schrödinger operators and its applications, Diffusion Processes and Related Problems in Analysis, Birkhäuser, Boston, Mass., 1990, pp. 245-260.

Department of Mathematics, University of Missouri, Columbia, Missouri 65211

E-mail address: mathzz@mizzou 1 